\title{
Effects of hints and interpolated activity on solution of an insight problem*
}

\author{
ROGER L. DOMINOWSKI and REGINA JENRICK \\ University of Illinois at Chicago Circle, Chicago, Ill. 60680
}

Two experiments were conducted to determine the effects of hints and interpolated activity on Ss' behavior in trying to solve the hatrack problem. Results indicated that the "ceiling hint" was more effective in facilitating solution than the "clamp hint" but that interpolated activity alone was ineffective. In both experiments, Ss achieving low scores on the Gestalt transformations test responded to a hint more rapidly when it was given during continuous work compared to giving the hint after a period of interpolated activity; in contrast, high scorers responded more rapidly to a hint given after interpolated activity. Discussion centered on the conditions under which positive effects of interpolated activity might be expected.

An idea with considerable intuitive appeal is the notion of an "incubation" effect, namely, that a person who has worked on a problem without success will be aided by engaging in an interpolated activity for a period of time, then returning to the problem. However, early research on this question yielded contradictory results (see Woodworth \& Schlosberg, 1954). Recently, Murray \& Denny (1969) proposed an explanation for this pattern of findings. These investigators suggested that a period of interpolated activity impairs the performance of Ss of high ability but aids those of low ability. The rationale is that high-ability $\mathrm{Ss}$ are working productively at the time of interpolation and thus suffer through the disruption of these productive efforts. In contrast, low-ability Ss are presumably fixated in stereotyped responses to the problem; thus the interpolation weakens this fixation and allows them to adopt more fruitful approaches to the problem. Murray and Denny reported findings in support of this analysis.

A weakness of the Murray and Denny finding was the failure to obtain a main effect of problem-solving ability. In that study, ability was identified with scores on the Gestalt transformation test (French, Ekstrom, \& Price, 1963). The failure to find an overall relationship between test scores and performance on the problem agrees with the findings of Burke \& Maier (1965); these results lead to some doubt concerning the validity of the Gestalt transformation test (GTT) as a measure of problem-solving ability. Since the proposed explanation would

*The authors are indebted to C. Gill, D. Ignatov, S. Meyer, $M$. Pickel, W. Schiller, $R$. Vaicaitis, P. Walsh, O. Wasyliw, and $E$. Williams for their assistance in this study. have considerable importance for the development of a thorough account of human problem solving, it is critical that its empirical support be substantial. The present investigation was designed primarily as a constructive replication of the Murray \& Denny (1969) study.

The present study also involved giving a hint during problem solving, since one of the possible effects of giving a hint is to interrupt ongoing activity (Burke, Maier, \& Hoffman, 1966). On this basis, it might be expected that a hint would have different effects on Ss of high and low ability. Giving a hint would disrupt the productive activity of high-ability Ss but would interrupt unproductive activity for low-ability Ss. Of course, hints also provide information, suggesting certain directions and eliminating others, and it can be plausibly argued that high-ability Ss would be better able to use this information. Therefore, it is difficult to develop firm expectations concerning possible differential effects of hints for Ss of high and low ability. One possibility suggested, however, is that high-ability Ss should find a hint more beneficial when given after interpolated activity rather than during continuous work. In the latter case, the hint both disrupts ongoing activity and provides potentially useful information; when given after interpolated activity, upon return to the problem, the hint's disruptive effect is minimized and its facilitative effects might be more in evidence. EXPERIMENT 1

Design and Subjects

The experimental design was a 2 by 2 by 2 factorial combination of the variables: hint vs no hint, interpolated activity vs continuous work, and high vs low ability (GTT scores). Seventy-nine introductory psychology students fulfilling a course requirement were assigned to the experimental conditions in alternation. Since ability measures were obtained after the problem-solving experience, the number of Ss per cell was not constant, varying between 7 and 12 . The ability measure, GTT score, was based on 20 items, each of which requires $\mathbf{S}$ to choose an object having a part best suited to a specified function, for example, "From which of the following would you make a hose: a tree, a cigarette, a shirt, a bicycle, or eyeglasses?" The test was administered in various-sized groups from 1 to 3 weeks after the Ss had worked on the problem.

Problem and Instructions

The problem used was Maier's hatrack problem, which requires $S$ to contruct a stable hatrack using two boards and a $\mathrm{C}$ clamp. The correct solution consists of wedging the two boards between the ceiling and the floor, holding them in place with the $\mathrm{C}$ clamp, with the clamp also serving as a hook. The boards used were 1 in. $x$ 2 in. $x 4 \mathrm{ft} 5$ in. and 1 in. $x 2$ in. $x 5 \mathrm{ft}$ 9 in.; a 2-in. C clamp was provided. The experimental room was approximately $12 \times 10 \times 10 \mathrm{ft}$. Because the room was carpeted, a possible solution consisted of propping one board against the wall. To eliminate this possibility, a circle large enough to contain either board when laid on the floor was drawn in the center of the room and instructions indicated that the construction had to be contained in the circle.

The basic instructions were as follows: "Your problem will be to construct a hatrack using those two boards and the $C$ clamp. The hatrack must be contained entirely inside the circle, i.e., there can't be any boards sticking out anywhere, and it must be strong enough to support a coat, i.e., it cannot be balanced in some flimsy way-it must be quite sturdy." Differential information was given on the time allowed.

\section{Procedure}

Each $S$ was seen individually and was allowed a maximum of $15 \mathrm{~min}$ working time. In the continuous work (C) condition, $\mathrm{S}$ was allowed to work on the problem for up to $15 \mathrm{~min}$ without interruption. In the hint $(H)$ condition, the ceiling hint (Burke et al, 1966) was given after 5 min of work; this hint is simply the statement, "The ceiling is part of the solution." In the interpolated-activity (I) condition, S was stopped after 5 min and taken to the next room to perform a different task of $5 \mathrm{~min}$ duration, then returned to the problem with up to $10 \mathrm{~min}$ allowed. In the hint plus interpolated activity (HI) condition, the ceiling hint was given when $S$ returmed to the problem after the interpolated 
Table 1

Solution Percentages and Mean Solution Times (Minutes) for Ss of High and Low Ablity in Each Condition

\begin{tabular}{llcrrr}
\hline & & \multicolumn{3}{c}{ Conditions } \\
\cline { 3 - 6 } & & C & I & H & HI \\
\hline High & Percent & 30 & 50 & 100 & 100 \\
Ability & Mean & - & - & 1.68 & 1.25 \\
Low & Percent & 12 & 8 & 100 & 83 \\
Ability & Mean & - & - & 0.66 & 3.52 \\
\hline
\end{tabular}

Note-Solution times were measured from the point at which the hint was given.

activity. In the conditions in which interpolated activity was given, half the Ss were given a free-association task, while the other half worked on an anagram task requiring the production of as many words as possible from the letters in two stimulus words, presented separately. Since these two subgroups did not differ, they are not distinguished in the following discussion.

The problem was considered solved when $\mathrm{S}$ wedged the boards between the floor and ceiling and began to hold them in place with the clamp (completion of the structure was not required). For Ss who failed, the solution was demonstrated at the end of the session. Three Ss solved the problem during the first $5 \mathrm{~min}$ and were excluded from the analysis; those given interpolated activity reported that they did not attempt to work on the problem during that activity.

Results and Discussion

High and low ability were defined as GTT scores of 11 or above and 10 or below, respectively, which was roughly a median split for the entire sample. The percentage of solutions in each group is indicated in Table 1 , where it can be seen that the ceiling hint markedly increased the likelihood of solution. For Conditions $\mathrm{H}$ and HI, $96 \%$ of the Ss solved the problem, whereas only $25 \%$ solved in Conditions $C$ and $\mathrm{I}(\mathrm{z}=6.20, \mathrm{p}<.001)$. This finding agrees with earlier results (Burke, 1969; Burke et al, 1966; Maier \& Burke, 1967). Although there was a numerical difference between solution percentages of high-and low-ability $S_{s}$ in Conditions $\mathbf{C}$ and $\mathbf{I}$, this difference was unreliable $(z=1.07)$.

The poor performance in Conditions $C$ and I precluded calculation of solution times for these groups. The solution times for Conditions $\mathrm{H}$ and $\mathrm{HI}$ were subjected to an unweighted means analysis of variance (Myers, 1966) after a square-root transformation was applied to reduce heterogeneity of variance. As indicated by the means given in Table 1 , the timing of the hint had different effects for Ss of high and low ability $[\mathrm{F}(1,34)=4.82, \mathrm{p}<.05]$. For Ss of high ability, the hint produced slightly faster solutions when given after a period of interpolated activity, while low-ability Ss made better use of the hint when it was given during continuous work. The interaction was produced primarily by the relatively long solution times for low-ability Ss when given the hint after interpolated activity. This result suggests that these Ss require a certain "warm-up" period before being able to utilize the information, although there is no direct evidence on this point.

A major outcome of the experiment was the failure to observe any effects of interpolation alone, contrary to the results of the study by Murray \& Denny (1969). Since the problem proved quite difficult, it is possible that interpolated activity differentially affected the behavior of high- and low-ability Ss, but not in a way which affected solution probability. To test this hypothesis, a second experiment was conducted.

\section{EXPERIMENT 2}

The major purpose of this experiment was to obtain a record of each S's solution attempts in order to determine (1) whether high- and low-ability Ss behave differently in the problem situation, (2) if interpolated activity affects the solution attempts Ss make, and (3) whether the effect of interpolated activity differs for the two $S$ types. The general design of the experiment was like that of Experiment 1; since the effect of the ceiling hint seemed clearly demonstrated, a different hint, the "clamp hint," was employed in this experiment. The clamp hint ("In the correct solution, the coat would hang from the clamp") has proved less effective than the ceiling hint in facilitating solution (Burke, 1969; Burke et al, 1966; Maier \& Burke, 1967).

\section{Design and Subjects}

The factorial design consisted of combinations of hint vs no hint, continuous work vs interpolated activity, and high vs low ability (GTT scores). As a course requirement, 62 introductory psychology students served as Ss and were assigned to experimental condition in alternation. Since GTT scores were obtained after the problem-solving experience, the number of Ss per cell was not constant. The test was administered to each $\mathrm{S}$ immediately after the problem session.

\section{Procedure}

The hatrack problem was again used under the same general conditions. Each $\mathbf{S}$ was seen individually and was allowed a maximum of $13 \mathrm{~min}$ working time. Condition $\mathrm{C}$ allowed up to $13 \mathrm{~min}$ work without interruption; in Condition $\mathrm{H}$, the clamp hint was given after 3 min work. In Conditions $I$ and HI, Ss were stopped after $3 \mathrm{~min}$ work and administered an anagram task of $3 \mathrm{~min}$ duration in another room and then returned to the problem, with the clamp hint given upon return in Condition HI.

The basic instructions were similar to those used earlier, emphasizing that the rack must be sturdy and contained entirely within the circle. The $S$ was told to try to solve the problem as quickly as he could; the distribution of working time was not indicated, and Ss receiving interpolated activity were not told that they would return to the problem. Subsequent questioning of these Ss indicated that they did not expect to return.

Results and Discussion

High ability was defined as GTT scores of 10 or above $(N=32)$, with low ability consisting of scores of 9 or less $(N=30)$; the number of $S s$ in individual cells of the design varied between 6 and 10. Analyses of variance were conducted using the method of unweighted means (Myers, 1966). The problem proved quite difficult, with only four solutions produced by the entire group of Ss. In contrast to the obvious facilitation produced by the ceiling hint in Experiment 1, the clamp hint failed to affect solution probability. This finding is in agreement with earlier results showing the clamp hint to facilitate solution only after a relatively long working time, (i.e., 20 min; Burke et al, 1966). However, the clamp hint did affect the nature of solution attempts; $97 \%$ of solution attempts made immediately after the clamp hint was given were balance solutions (leaning the boards against each other in a "X" or "T" shape with the clamp joining them), whereas this was true of only $67 \%$ of comparable solution attempts when the hint was not given $\left[x^{2}(1)=7.13, p<.01\right]$. Thus, the results support the position of Burke et al (1966) that the clamp hint does change direction in problem solving but tends to lead a different incorrect attempt rather than to the solution.

Protocols were also examined to determine if solution attempts varied with the ability level of the Ss or as a function of interpolated activity. In 
the present study, in addition to the balance solution, the other type of incorrect attempt which occurred was the base solution (aligning the boards in an inverted " $\mathrm{T}$ " structure with the clamp joining them at the floor level). At the outset of the problem, there was a slight tendency for base solutions to be tried more by high-ability Ss (33\%) than by low-ability Ss $(11 \%)\left[x^{2}(1)=3.07\right.$, $p<.10]$; the two groups did not differ in terms of either latency or duration of initial attempts. However, the duration of the initial solution attempt was longer for those Ss who tried balance solutions (mean = $119 \mathrm{sec})$ than for those trying base solutions $($ mean $=52 \mathrm{sec}) \cdot[F(1,57)=$ $63.63, p<.001]$. This was in direct contrast to Burke's (1970) finding that base solutions had the longest durations of any incorrect solution attempts. Although there is no ready explanation for the difference in results, it must be noted that the experimental situations and methods of analysis differed in the two studies.

The rate at which overt solutions were attempted was higher during the first $3 \mathrm{~min}$ working time (mean $=.70$ attempts/min), before the introduction of differential treatments, than it was in the last $10 \mathrm{~min}$ (mean $=.54$ attempts $/ \mathrm{min}$ ) $[F(1,57)=10.81, p<.01]$. However this change was unrelated either to ability level or to the various experimental manipulations. The overall reduction in the rate of production of attempted solutions is in agreement with Duncan's (1962) finding.

The introduction of interpolated activity alone did not affect the likelihood of changing the type of solution attempted. The first solution attempted after interpolated activity was the same as the last attempt prior to the interval in $73 \%$ of the cases; in the continuous work condition, $50 \%$ of successive solutions were the same at the comparable point in time. While these percentages were not significantly different $\left(x^{2}=1.2\right)$, there is obviously no support for the notion that interpolated activity facilitates the adoption of a new approach to the problem.

In Experiment 1, interpolated activity and ability level yielded an interaction when solution latencies after the ceiling hint were analyzed. A comparable analysis was made for Experiment 2, using latencies of first posthint solution attempts, which were virtually always incorrect balance solutions. For high-ability Ss, the mean latencies were $3.67 \mathrm{~min}$ for Condition $\mathrm{H}, \quad 1.14 \mathrm{~min}$ for Condition $\mathrm{HI}$; the comparable means for low-ability Ss were $0.38 \mathrm{~min}$ and $0.80 \mathrm{~min}$, respectively. The analysis indicated a main effect for ability level $[F(1,27)=5.70, p<.025]$, and there was a suggestion of an interaction between ability level and interpolated activity $[F(1,27)=3.74, p<.10]$. The pattern of means was the same in the two experiments, with high-ability Ss responding faster to the hint when it was given after interpolated activity, while the reverse was true for low-ability Ss. However, it must be noted that the interaction of Experiment 1 reflected primarily the long latencies for low-ability $S$ s in Condition HI, whereas the interaction in Experiment 2 was produced largely by the long latencies for high-ability Ss in Condition $\mathrm{H}$.

\section{GENER AL DISCUSSION}

The present results were consistent with previous research in several aspects: the greater effectiveness of the ceiling hint in facilitating solution of the problem, as compared to the clamp hint (e.g., Burke et al, 1966); the decrease in rate of solution attempts over time (Duncan, 1962); and the lack of relationship between scores on the Gestalt transformations test and problem-solving efficiency (Burke \& Maier, 1965; Murray \& Denny, 1969). However, a major result of the present study was the failure to replicate Murray and Denny's finding of different effects of interpolated activity alone on the performance of Ss of high and low ability (GTT scores).

Murray and Denny suggested that high scorers are more active and orderly problem solvers than low scorers and that, consequently, interpolated activity disrupts productive efforts for high scorers while disrupting inappropriate fixation for low scorers. Their analysis is supported by the present results only insofar as it provides a plausible explanation of the differential effects of giving a hint during continuous work vs after interpolated activity. In particular, the finding that high scorers respond more slowly to a hint given during continuous work is consistent with their position. If these Ss are actively pursuing a particular problem-solving direction, this direction must be overcome before the hint can be utilized. In contrast, when the hint is given after interpolated activity, when the Ss are not currently active, the hint occurs in a more neutral context and may thus be utilized more rapidly. However, this analysis must be considered tentative in view of the lack of evidence indicating that the Gestalt transformations test is a meaningful measure of ability for such problems.

The inconsistency of results regarding the effects of interpolated activity alone seems most likely to be due to variation in the nature of the problem used. It is possible that the hatrack problem represents a type of task for which interpolated activity will have little effect. Success on this task depends essentially on a single idea, discovering that the ceiling, which is not explicitly mentioned in the statement of the problem, is part of the solution. Furthermore, only a small number of different directions is possible, thus making it difficult to continue the generation of new and different approaches for any extended period of time. Most of the evidence indicating positive effects of interpolated activity involves work on problems which are difficult for the problem solver (as Murray and Denny's analysis requires) and which, perhaps more importantly, involve finding the correct relationships among a number of component ideas (see Woodworth \& Schlosberg, 1954). The task used by Murray and Denny, although resembling the hatrack problem in some respects, nevertheless differed in that it required finding the right arrangement of a number of objects, all of which were given and which could be combined in several different ways. For this task, $S$ was restricted to an area behind a line on the floor and, with a nail, a pair of pliers, a length of string, a pulley, some elastic bands, and several newspapers at his disposal, was required to transfer several steel balls from a drinking glass to a metal cylinder, both of which were $8 \mathrm{ft}$ from the line and at the floor level. It seems likely that studying the effects of interpolated activity with a variety of problems selected to represent different levels of complexity might prove fruitful.

Another factor deserving attention is the length of time Ss work on a problem prior to interpolated activity. Positive effects of a "rest interval" are expected only when $S$ has "exhausted" his supply of relevant ideas or has fixated on a particular inappropriate direction. The average amount of time required for this state to be reached seems likely to vary with the task as well as with the ability level of the Ss. Consequently, a coherent pattern of results might be expected if research includes parametric study of the length of the initial working period. Similar considerations might apply to the duration of the interpolated activity itself, although this seems less likely since the results to date suggest that interpolated activity of very brief duration provides a sufficient break in problem-solving activity.

\section{REFERENCES}

BURKE, R. J. A comparison of two properties of hints in individual problem solving. Joumal of General Psychology, $1969,81,3-21$

BURKE, $R$. J. Nearsuccess and solution 
persistence in individual problem solving. Journal of General Psy chology, 1970, 82, $133-138$.

BURKE, R. J., \& MAIER, N. R. F Attempts to predict success on an insight problem. Psychological Reports, 1965. $17,303-310$.

BURKE, R. J., MAIER, N. R. F, \& HOFFMAN, L. R. Functions of hints in individual problem solving. American Journal of Psychology, 1966, 79 . 389-399.
DUNCAN, C, P. Probability vs. latency of solution of an insight problem. Psychological Reports, 1962, 10, 119-121.

FRENCH, J. W., EKSTROM, R. B., \& PRICE, L. A. Kit of reference tests for cognitive factors. Princeton: Education Testing Service, 1963.

MAIER, N. R. F., \& BURKE, R. J. Influence of timing of hints on their effectiveness in problem solving. Psychological Reports, 1967, 20, 3-8.
MURRAY, H. G., \& DENNY, J. P. Interaction of ability level and interpolated activity (opportunity for incubation) in human problem solving. Psychological Reports, 1969, 24, 271-276.

MYERS, J. L. Fundamentals of experimental design. Boston: Allyn \& Bacon, 1966.

WOODWORTH, R. S. \& SCHLOSBERG, $H$. Experimental psychology. (Rev. ed.) New York: Holt, 1954. 